\title{
Estudio de la opinión del profesorado de la Universitat de Barcelona de la nueva herramienta de descubrimiento del CRAI, Cercabib
}

\author{
Andreu Sulé ${ }^{1}$, Juanjo Boté ${ }^{2}$, Marina Salse ${ }^{3}$ \\ ${ }^{1}$ 0000-0002-2467-3678 + Departament de Biblioteconomia, Documentació i Comunicació \\ Audiovisual \& Centre de Recerca en Informació, Comunicació i Cultura. Universitat de Barcelona. \\ C/ Melcior de Palau 140,08014 ES, Barcelona. sule@ub.edu. \\ ${ }^{2}$ 0000-0001-9815-6190 + Departament de Biblioteconomia, Documentació i Comunicació \\ Audiovisual \& Centre de Recerca en Informació, Comunicació i Cultura. Universitat de Barcelona. \\ C/ Melcior de Palau 140,08014 ES,Barcelona.juanjo.botev@ub.edu. \\ ${ }^{3}$ 0000-0003-2003-7225 + Departament de Biblioteconomia, Documentació i Comunicació \\ Audiovisual \& Centre de Recerca en Informació, Comunicació i Cultura. Universitat de Barcelona. \\ C/ Melcior de Palau 140, 08014 ES, Barcelona. salse@ub.edu.
}

Tipo de contribución: comunicación

Palabras clave: Herramientas de descubrimiento; Cercabib; Estudio de usuarios; Profesorado; Universitat de Barcelona; CRAI.

\section{Introducción}

En los últimos años, las herramientas de descubrimiento están sustituyendo los antiguos OPAC en buena parte de las bibliotecas del mundo, incluyendo las de nuestro país. Las dos principales diferencias de estas herramientas en comparación con los antiguos catálogos (OPAC) son la asimilación del modelo de búsqueda de Google (priorización de la búsqueda simple en una única casilla sin limitadores de campo ni operadores booleanos explícitos) y la recuperación de resultados en todos los fondos de la biblioteca, independientemente de su naturaleza (analógica o digital) y de su procedencia (colección propia, bases de datos, revistas electrónicas, repositorios, etc.). El objetivo final de las herramientas de descubrimiento es reproducir, en la medida de lo posible, la exitosa experiencia de usuario que ofrece Google, tratando de simplificar al máximo el proceso de búsqueda, por medio de una casilla única, de todos los recursos bibliográficos adquiridos y contratados por la biblioteca (ÁVILA-GARCÍA; ORTIZ-REPISO; RODRÍGUEZ-MATEOS, 2015).

En el caso del Centre de Recursos per a l'Aprenentatge i la Investigació (CRAI) de la Universitat de Barcelona (http://crai.ub.edu/) el cambio de sistema se produjo el 2018 cuando Cercabib (http://Cercabib.ub.edu/iii/encore/) sustituyó los anteriores sistemas de recuperación de información de la UB, fusionando en un único entorno de búsqueda el Catàleg de les biblioteques (https://cataleg.ub.edu/) y el ReCercador+ (http://mlplus.hosted.exlibrisgroup.com/primo library/libweb/action/search.do), y convirtiéndose en la ventanilla única de búsqueda simultánea de todos los fondos del CRAI, independientemente del

\footnotetext{
1 Cercabib es el nombre que la Universitat de Barcelona ha puesto a la solución Encore-Duet de Innovative (https://www.iii.com/). Esta solución consiste en una capa de interfaces que permite consultar simultáneamente los registros bibliográficos de un OPAC y los recursos electrónicos configurados en una plataforma de EBSCO Discovery Service (EDS).
} 
soporte, tipología o ubicación del recurso. Tal y como se expone en la página de presentación de Cercabib, con esta herramienta se pueden "realizar búsquedas de manera simultánea en todos los fondos del CRAI al margen del soporte, tipología o ubicación del recurso. Es decir: libros, revistas, artículos de revista, tesis, audiovisuales, entre otros; independientemente de si son recursos en papel o formato electrónico y de si están ubicados físicamente en alguno de nuestros CRAI Bibliotecas o alojados en un servidor propio o ajeno. En conjunción con el SIRE (Servicio Intermediario de acceso a los Recursos Electrónicos), el Cercabib os dará acceso a los recursos electrónicos subscritos por el CRAI." (http://crai.ub.edu/es/recursos-de-informacion/Cercabib).

Ahora bien, más allá de las declaraciones formales, el éxito de una herramienta de búsqueda se mide por la mayor o menor satisfacción de sus usuarios. Como en cualquier proceso de cambio, la implementación de una nueva herramienta de descubrimiento puede generar diversidad de opiniones entre sus usuarios. La introducción de nuevas interfaces gráficas, de nuevas funcionalidades y de una nueva filosofía de búsqueda basada en la consulta simultánea de todos los fondos del CRAI puede satisfacer a una parte de ellos, pero también puede generar reacciones adversas, ya sea por el propio hecho del cambio (la incomodidad de tener que aprender algo nuevo), por el desconocimiento de la herramienta o por posibles carencias o errores en el diseño del sistema.

Es por ello que el objetivo del presente trabajo es conocer la opinión, la experiencia de los usuarios de Cercabib, y más concretamente del profesorado de la Universitat de Barcelona (UB). Conocer sus opiniones ha de servir para mejorar el servicio ofrecido por el CRAI, ya sea por medio de campañas de difusión y formación orientadas a necesidades no detectadas inicialmente o a través de la modificación de aquellos elementos de información o de acción del buscador identificados por los usuarios como problemáticos. Además, puesto que el perfil y las necesidades de los profesores de la Universitat de Barcelona son similares a las de otros centros académicos del mundo, tanto la metodología del estudio como sus conclusiones pueden ser de utilidad para otras bibliotecas universitarias que recientemente también hayan sustituido su catálogo por una nueva herramienta de descubrimiento.

Este estudio forma pare de un proyecto más amplio de evaluación de Cercabib que se irá completando con el estudio de la experiencia de otros colectivos universitarios (estudiantes, bibliotecarios, etc.), así como con la evaluación de funcionalidades específicas de la herramienta (búsqueda simple, búsqueda avanzada, limitadores, facetas, etc.), de sus interfaces gráficas y del uso del fondo por medio de logs y estadísticas de préstamo.

\section{Metodología}

Para recoger la opinión y la experiencia de los profesores de la UB sobre Cercabib hemos optado por la entrevista personal basada en un cuestionario previo (Anexo). Ello nos ha permitido definir a los entrevistados con mayor precisión el objetivo del estudio, y también aclarar posibles dudas sobre el significado de las preguntas. Además, la entrevista permite la modulación de las preguntas adaptándolas al perfil del entrevistado e incluso derivar la conversación a otros aspectos relacionados con el objetivo del trabajo que ayudan a una mejor interpretación de los resultados.

Debido a este carácter cualitativo de nuestro estudio, el muestreo ha sido no probabilístico intencional (GONZÁLEZ-TERUEL; BARRIOS CERREJÓN, 2012), es decir, hemos seleccionado el profesorado no de manera aleatoria sino en base a una serie de criterios que consideramos adecuados para nuestro objetivo: 
- Profesorado con una antigüedad mínima de cinco años en la Universitat de Barcelona. Con ello queremos asegurar una cierta experiencia en el uso de las herramientas de búsqueda del CRAI i especialmente del anterior Catàleg de les biblioteques.

- Paridad de profesorado dedicado principalmente a actividad docente y profesorado dedicado principalmente a actividad investigadora. Con ello queremos detectar posibles diferencias de opinión de Cercabib relacionadas con diferentes necesidades de uso de la información.

- Profesorado perteneciente a diferentes áreas y disciplinas. Para detectar posibles diferencias de opinión de Cercabib relacionadas con conocimientos, habilidades y necesidades de información de grupos de usuarios específicos.

- Paridad de profesorado por edad. Con ello queremos detectar posibles diferencias de opinión de Cercabib relacionadas con diferentes expectativas y experiencias de búsqueda de información.

Las entrevistas se han realizado de febrero a marzo de 2019. El número total de profesores entrevistados ha sido de 22 , cifra suficientemente representativa puesto que a partir de la veintena de encuentros ya pudimos constatar que habíamos llegado a la saturación de información que podían aportar las entrevistas, es decir, que los nuevos profesores dejaban de aportar datos adicionales o información relevante para el estudio de Cercabib.

El perfil de estos 22 profesores ordenado por disciplinas es el siguiente:

\begin{tabular}{|l|c|c|c|c|}
\hline \multicolumn{1}{|c|}{ Disciplina } & Antigüedad & \multirow{2}{*}{ Edad } & \multicolumn{2}{c|}{ Dedicación } \\
\cline { 3 - 5 } & & & $\%$ docente & \% investigadora \\
\hline Arqueología & 1990 & 56 & 50 & 50 \\
\hline Biblioteconomía y Documentación & 1987 & 59 & 80 & 20 \\
\hline Biblioteconomía y Documentación & 1994 & 57 & 62 & 38 \\
\hline Biblioteconomía y Documentación & 1995 & 51 & 50 & 50 \\
\hline Biblioteconomía y Documentación & 2002 & 43 & 86 & 15 \\
\hline Biblioteconomía y Documentación & 2002 & 46 & 50 & 50 \\
\hline Biblioteconomía y Documentación & 2007 & 49 & 40 & 60 \\
\hline Comunicación Audiovisual y Publicidad & 2010 & 47 & 50 & 50 \\
\hline Enfermería & 2007 & 60 & 80 & 20 \\
\hline Filosofia & 2003 & 43 & 50 & 50 \\
\hline Historia & 1977 & 68 & 40 & 60 \\
\hline Historia & 1981 & 64 & 50 & 15 \\
\hline Historia & 1986 & 63 & 50 & \\
\hline Historia & 1993 & 59 & 50 & 50 \\
\hline Historia & 1995 & 61 & & 50 \\
\hline Historia & 1996 & 58 & & 60 \\
\hline Historia & 1997 & 49 & 60 & 0 \\
\hline Historia & 2000 & 50 & 51 & 40 \\
\hline Historia & 2003 & 56 & 50 & 49 \\
\hline Interacción Persona-Computadora & 1998 & 50 & 75 & 50 \\
\hline Microbiología & 2012 & 37 & 53 & 25 \\
\hline Psicología & 1994 & 52 & 50 & 56 \\
\hline
\end{tabular}


Por disciplinas:

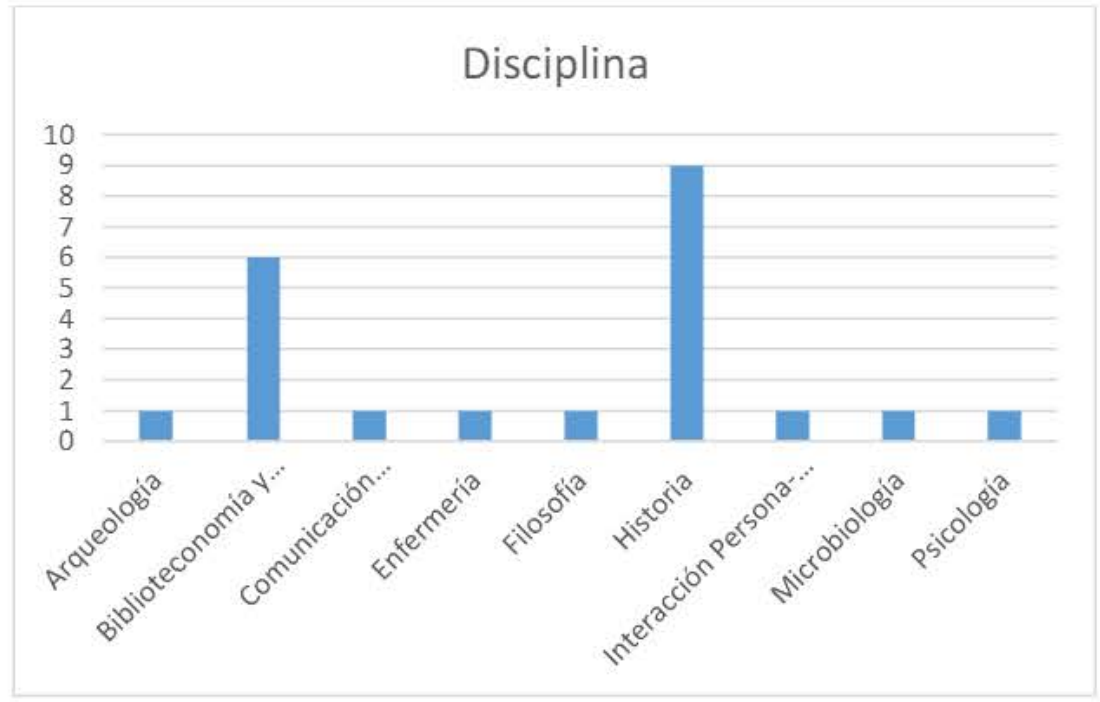

Por franjas de antigüedad en la Universitat de Barcelona:

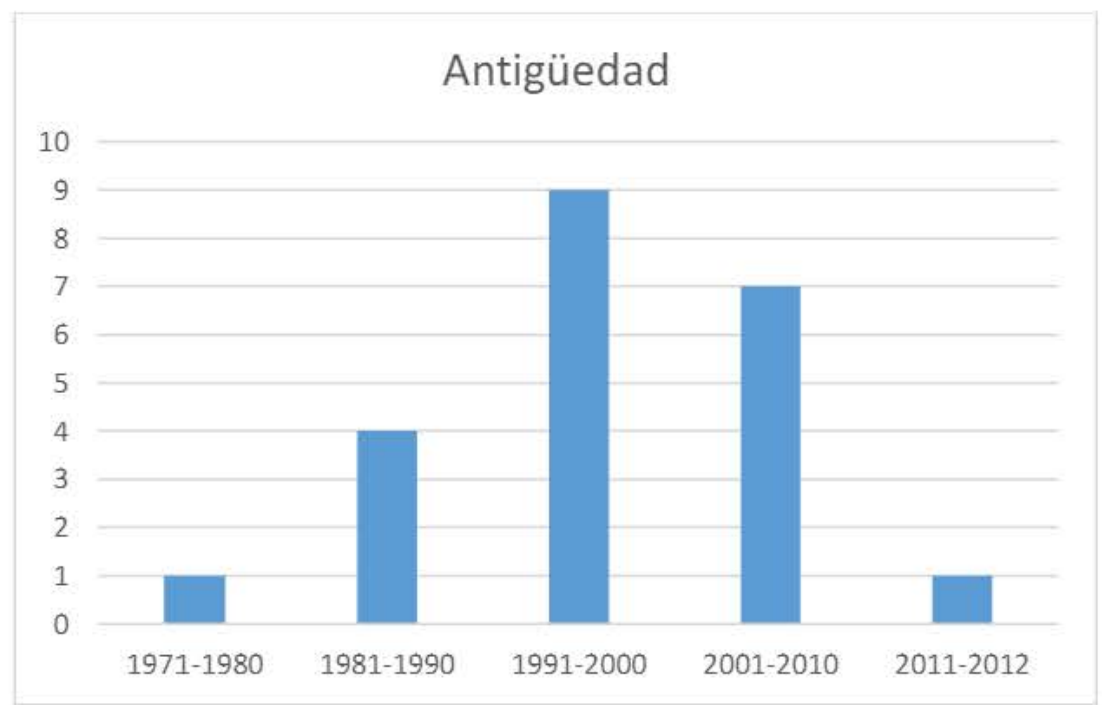

Por franjas de edades: 


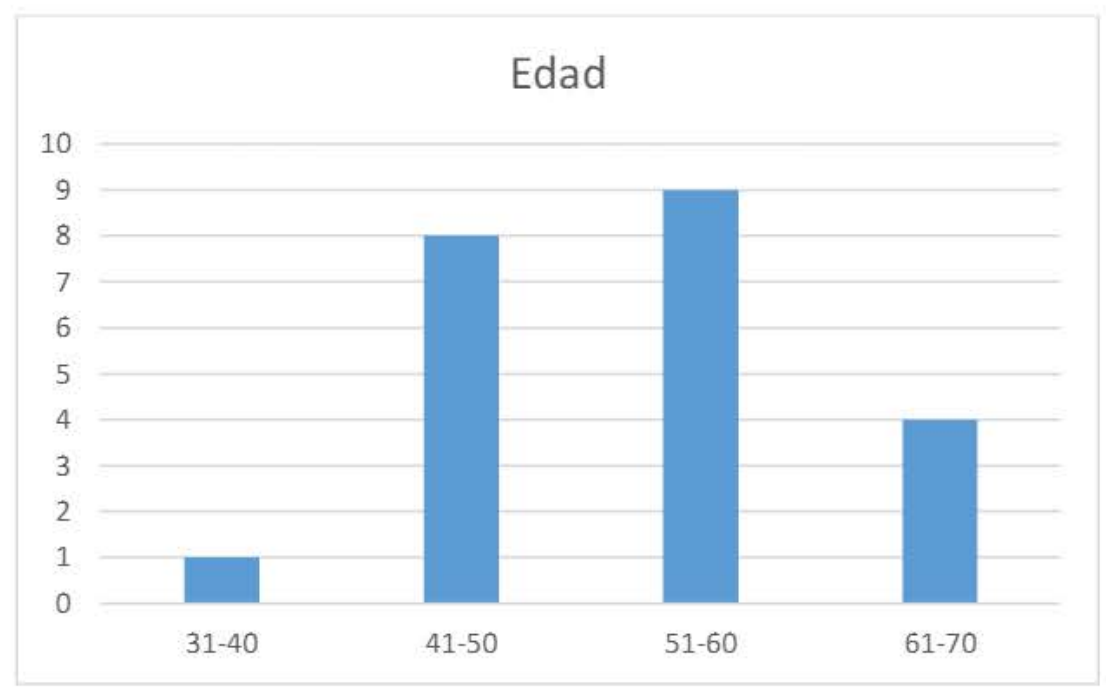

Por franjas de dedicación ("Doc" = Docente; "Inv" = Investigadora):

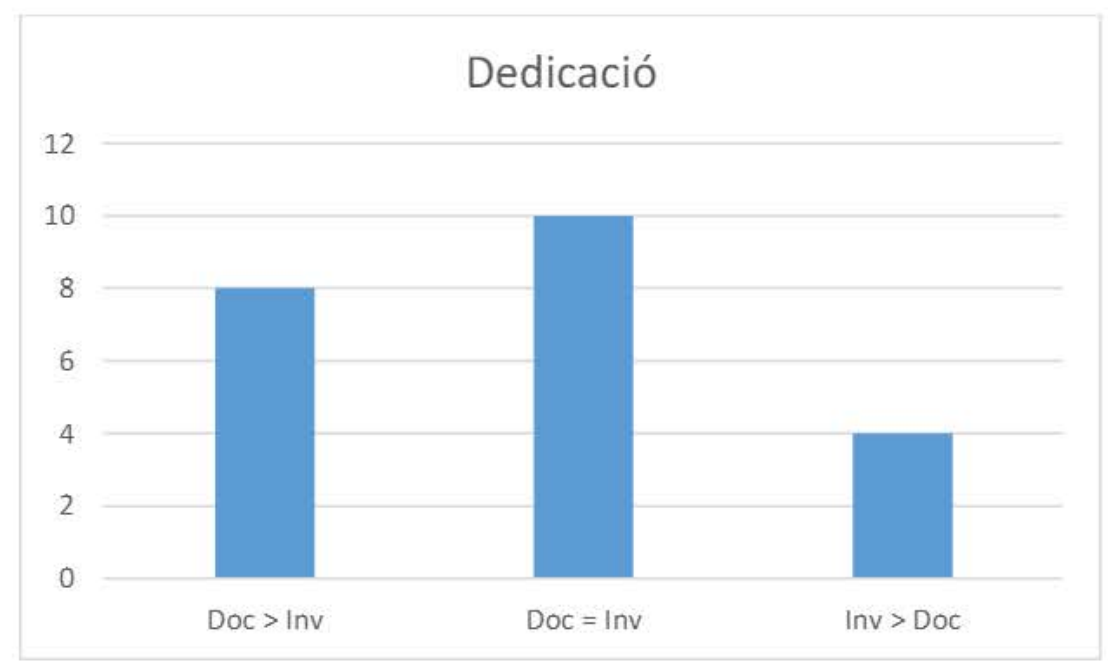

\section{Antecedentes}

La revisión bibliográfica muestra el interés en este tipo de estudios, si bien son escasos los trabajos centrados en el colectivo del profesorado. Tal y como indican NICHOLS et al. (2017), los estudiantes son el grupo de usuarios que más se ha tenido en consideración para la evaluación de las herramientas de descubrimiento y, de hecho, son los principales destinatarios de la formación de las bibliotecas en este tipo de sistemas. Y, aunque en menor medida, también son abundantes las investigaciones sobre la opinión y percepción de los bibliotecarios sobre las herramientas de descubrimiento.

Los estudios con estudiantes muestran que sus expectativas están fuertemente condicionadas por su experiencia con Google, Google Scholar y Amazon, tanto por lo que hace a la simplicidad de la búsqueda como a la gratificación instantánea (NICHOLS et al., 2017). DALAL, KIMURA y HOFMANN (2015) llevaron a cabo un estudio con estudiantes de la Rider University (Estados Unidos de América) donde observaron lo que puede considerarse una de las principales deficiencias de este tipo de servicio, a saber, el desconocimiento del alcance de la herramienta en tanto que no sabían que ésta une el catálogo, varias bases de datos y publicaciones académicas. De hecho, la mayoría de ellos desconocían las diferencias entre Google y la herramienta de descubrimiento. 
Las opiniones de los bibliotecarios recogidas por diferentes autores muestran críticas sobre cuestiones técnicas de las herramientas. FAWLEY y KRYSAK (2014) realizaron una encuesta a través de la lista de distribución ili-listserv, de la American Libraries Association, a la que respondieron 152 subscriptores. Se trataba de bibliotecarios universitarios que utilizaban una herramienta de descubrimiento (EBSCO Discovery Service, Summon de Serials Solutions y Primo de Ex Libris, principalmente) para la formación en alfabetización informacional. Los resultados mostraron una alta valoración de la herramienta $(76 \%)$ al considerarla un buen punto de partida para la investigación (especialmente en los estudios de nivel inferior) y por ofrecer resultados en formatos diferentes. Por su parte, los bibliotecarios que no valoraron bien la herramienta $(24 \%)$ criticaron la poca fiabilidad de la ordenación por relevancia, el abrumador número de resultados de búsqueda y la creación de falsas expectativas de acceso inmediato a todos los materiales. Preguntados por las mejoras que introducirían en el sistema, una de las más seleccionadas fue mejor comprensión de cómo la herramienta busca y ordena los resultados. En este punto vale la pena destacar el trabajo de AHARONY y PREBOR (2015), que contempla, entre otras valoraciones más técnicas, un estudio de personalidad orientado a descubrir por qué algunos bibliotecarios son más propensos a aceptar o a rechazar las herramientas de descubrimiento. Los investigadores realizaron una encuesta entre 125 bibliotecarios y profesionales de la información de Israel con al que descubrieron que las personas con una actitud más negativa ante la adopción de nuevas tecnologías tenían una visión más pesimista y una menor satisfacción con las herramientas de descubrimiento; por contra, aquellas personas que tenían un nivel de satisfacción más elevado eran aquellos que tenían una actitud abierta y positiva a la incorporación nuevas tecnologías.

Los estudios sobre la percepción de los profesores de las herramientas de descubrimiento son, como se ha comentado anteriormente, escasos. De hecho, algunos de ellos no son el producto de la opinión directa de los docentes sino de valoraciones realizadas por los bibliotecarios del centro. Por ejemplo, NICHOLS et al. (2017), en un estudio que recoge la opinión de 56 bibliotecarios referencistas y de formación de usuarios en universidades de los estados Unidos de América, constatan el poco predicamento que las herramientas de descubrimiento tienen entre los profesores. Únicamente en el $13 \%$ de las bibliotecas encuestadas la mayoría o todo el personal docente hacen uso las herramientas de descubrimiento; por el contrario, el mismo estudio identificó altas tasas de uso entre estudiantes y bibliotecarios (en el $55 \%$ de las bibliotecas).

WELLS y RICHARDSON (2014) llevaron a cabo un estudio sobre las expectativas de los usuarios de la Curtin University (Australia) de una instalación de la herramienta Primo implementada cinco años atrás. A diferencia de investigaciones sobre herramientas recién instaladas, el trabajo de WELLS y RICHARDSON tiene la particularidad de encuestar usuarios con experiencia en el manejo del sistema. Quizás por ello los resultados obtenidos mostraron una alta valoración de las características de Primo, en concreto su capacidad de integración de múltiples sistemas en el proceso de búsqueda, el fácil acceso a los recursos en línea, las opciones de filtrado y la cobertura del sistema. En definitiva, los usuarios valoraron Primo como una herramienta fácil de usar.

\section{Resultados del estudio}

El cuestionario que ha servido de guía para la realización de las entrevistas personales a los profesores de la UB consta de dos partes:

- Una primera para recoger la información demográfica del docente (fecha de nacimiento, año de inicio de la vinculación con la UB, dedicación, etc.); 
- Una segunda compuesta por 15 preguntas de respuesta abierta de carácter cualitativo sobre la actitud a los cambios tecnológicos, los hábitos de búsqueda de información y la parte más importante dedicada a la percepción y valoración de Cercabib.

A continuación se exponen de forma sumariada los resultados de las entrevistas agrupados en cinco preguntas clave:

- Conocimiento de la existencia de Cercabib

- Conocimiento del alcance de Cercabib

- Experiencia (positiva o negativa) en el uso de Cercabib

- Aspectos más positivos y más negativos de Cercabib

- Preferencia entre el catálogo anterior y Cercabib

\subsection{Conocimiento de la existencia de Cercabib}

El conocimiento de la existencia de Cercabib es muy amplio entre el profesorado entrevistado (86\%, 19 de 22). Algunos docentes han sido más concretos que otros en la denominación del sistema, pero casi todos ellos saben que el servicio de bibliotecas de la UB dispone de una nueva herramienta de búsqueda.

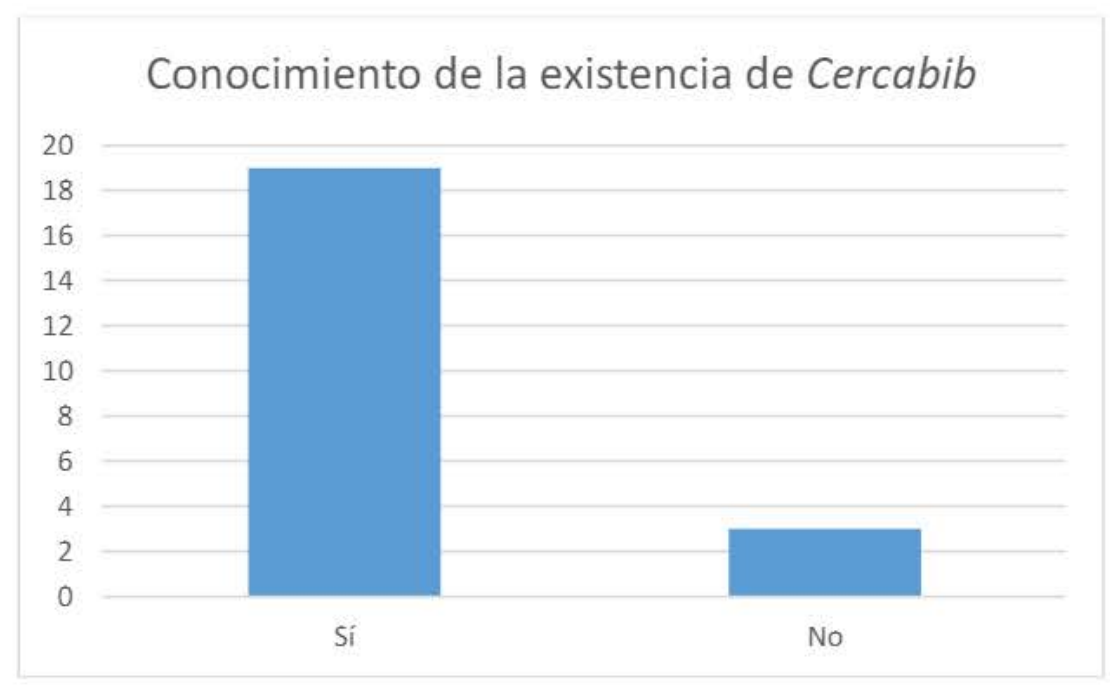

Segmentados los resultados por disciplina, no se observan diferencias significativas que permitan detectar un conocimiento específico de Cercabib derivado de la especialización temática de los entrevistados. 


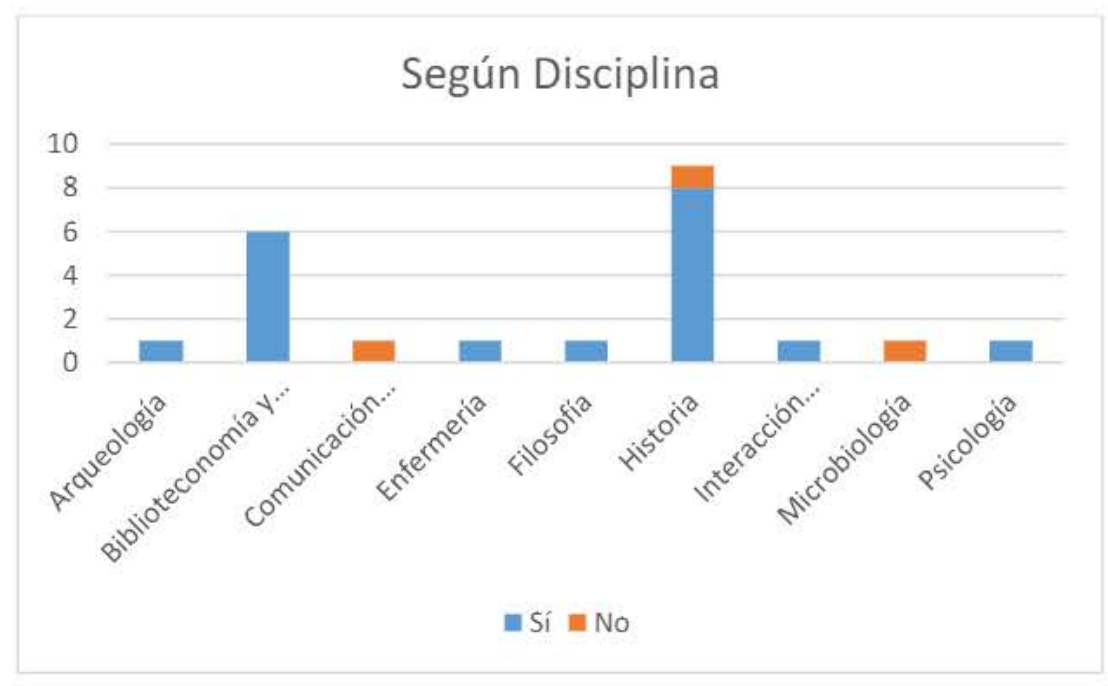

Por contra, en el caso de la antigüedad en la UB sí que se aprecia un menor conocimiento de Cercabib en las personas que llevan menos años vinculadas a la universidad (franja de incorporación 20002010 y especialmente 2011-2012). Una posible explicación es que estos docentes acostumbran a ser personas más jóvenes que en las entrevistas manifestaron su preferencia por el uso de herramientas alternativas a Cercabib para buscar información (Google, Google Scholar, Web of Science, Scopus, ResearchID, Academia, etc.).

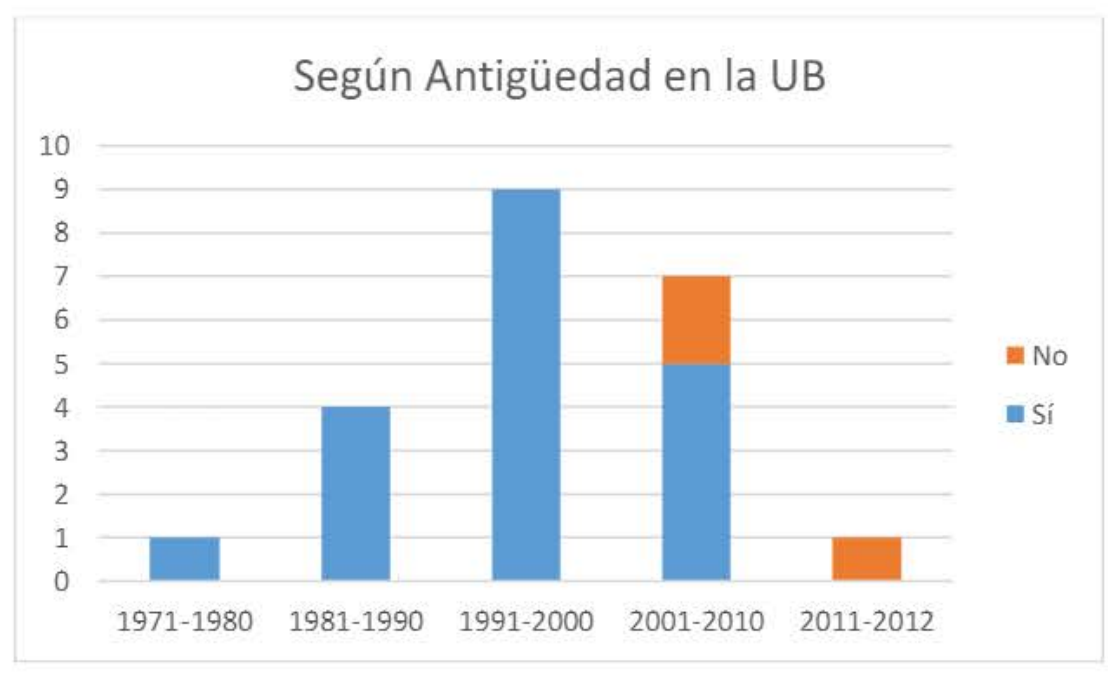

En la siguiente gráfica se puede observar que los resultados segmentados por edad ratifican la valoración hecha anteriormente: a medida que disminuye su edad los profesores tienen un menor conocimiento de la existencia de Cercabib. 


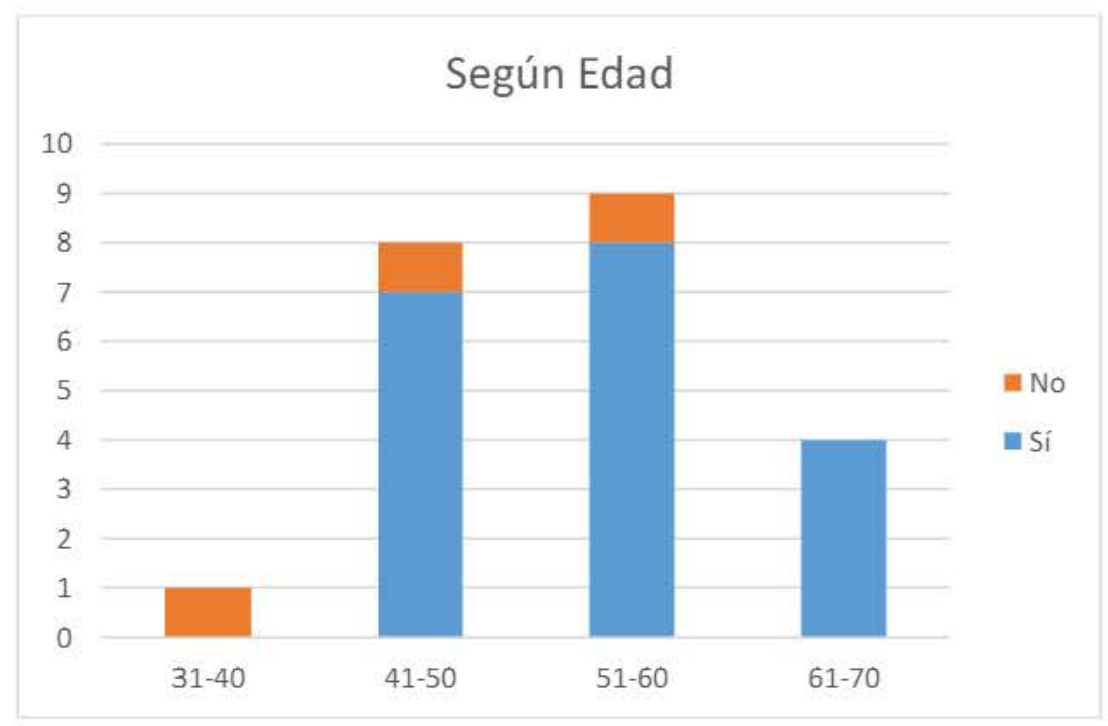

Por último, los datos segmentados por dedicación muestran una cierta tendencia al desconocimiento de Cercabib a medida que la docencia no es la actividad principal del profesorado. Este hecho es posible que esté relacionado con la preferencia por el uso de herramientas de búsqueda más específicas en los entornos de investigación. Algunos profesores manifestaron preferir buscar información en bases de datos bibliográficas directamente relacionadas con sus líneas de investigación.

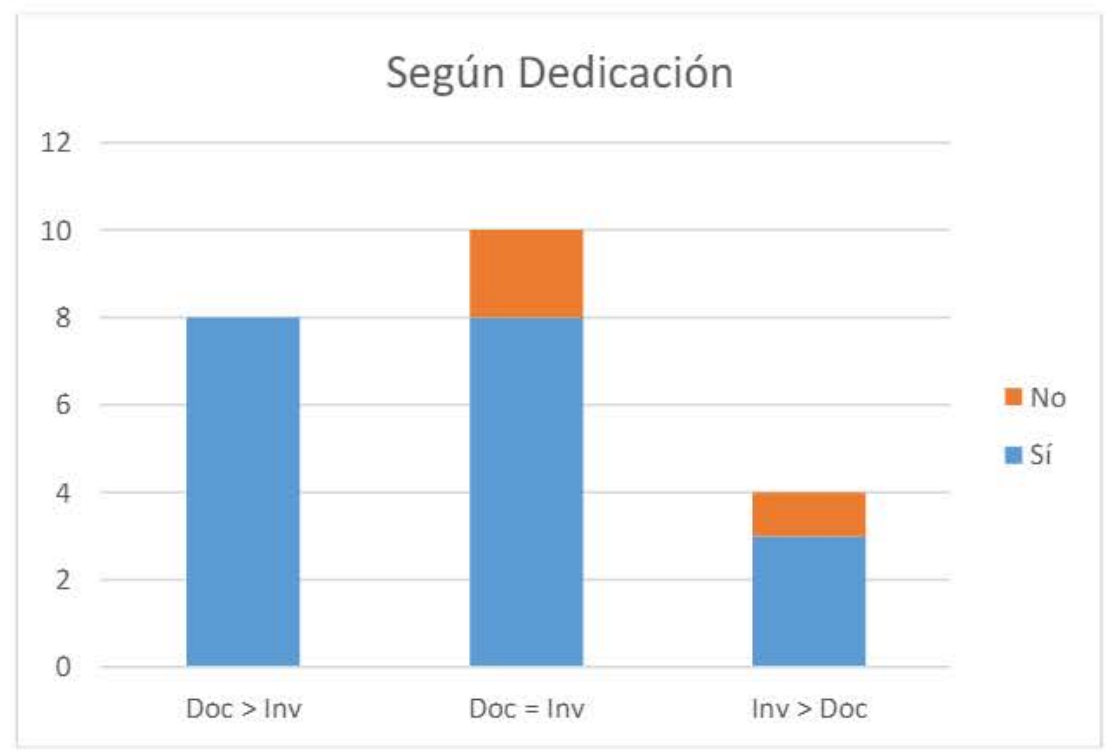

\subsection{Conocimiento del alcance de Cercabib}

Únicamente el $32 \%$ ( 7 de 22) de los profesores entrevistados manifestaron tener un conocimiento claro del alcance de Cercabib, es decir, qué tipos de documentos se pueden encontrar, de qué fuentes provienen o cuál es la diferencia en relación al anterior sistema de búsqueda del CRAI de la UB.

\begin{tabular}{|l|c|}
\hline \multicolumn{2}{|l|}{ Conocimiento sobre el alcance de Cercabib } \\
\hline No saben que pueden encontrar & 13 \\
\hline Facilitan una respuesta correcta & 7 \\
\hline
\end{tabular}




\subsection{Experiencia (positiva o negativa) en el uso de Cercabib}

La experiencia de uso de Cercabib es, en general, poco satisfactoria. Únicamente 6 de los 22 profesores entrevistados (27\%) manifestaron una valoración positiva de la herramienta. Del resto, 11 la valoraron negativamente y 5 no manifestaron ninguna opinión puesto que no empleaban Cercabib para buscar información.

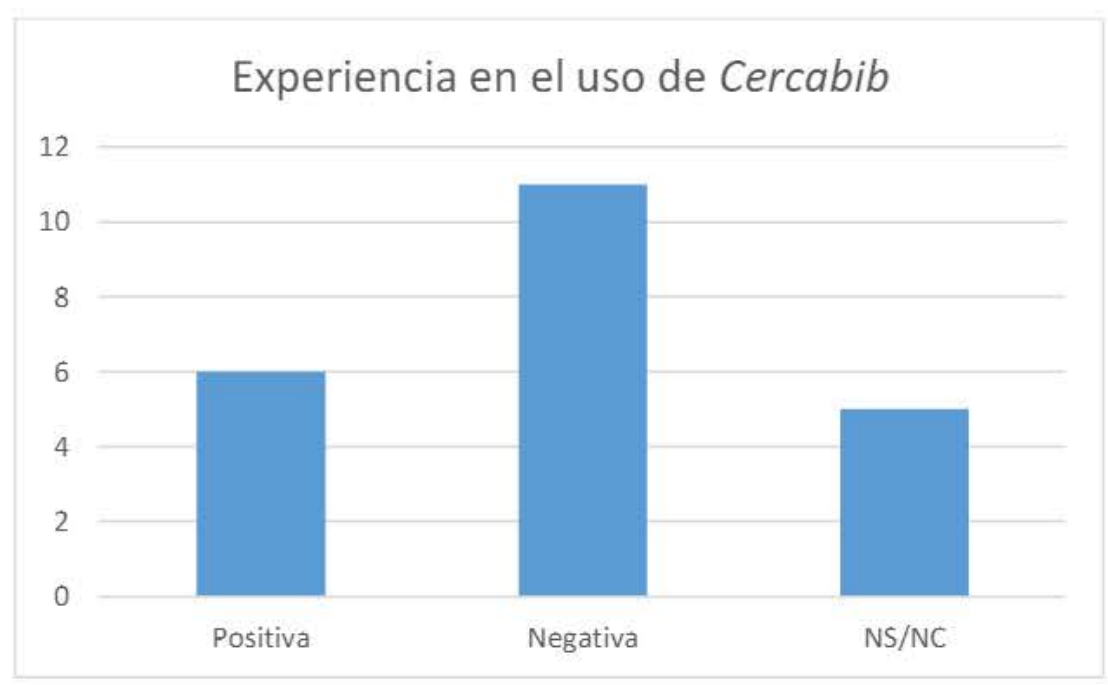

Si nos atenemos a la disciplina, se puede observar que los profesores de Biblioteconomía y Documentación son de los más críticos con Cercabib. Creemos que este hecho se debe a los conocimientos avanzados que estos docentes tienen de las herramientas de búsqueda, lo que les permite identificar como limitaciones o carencias del sistema cuestiones que otros docentes atribuyen a errores propios en el proceso de búsqueda (no saber buscar bien).

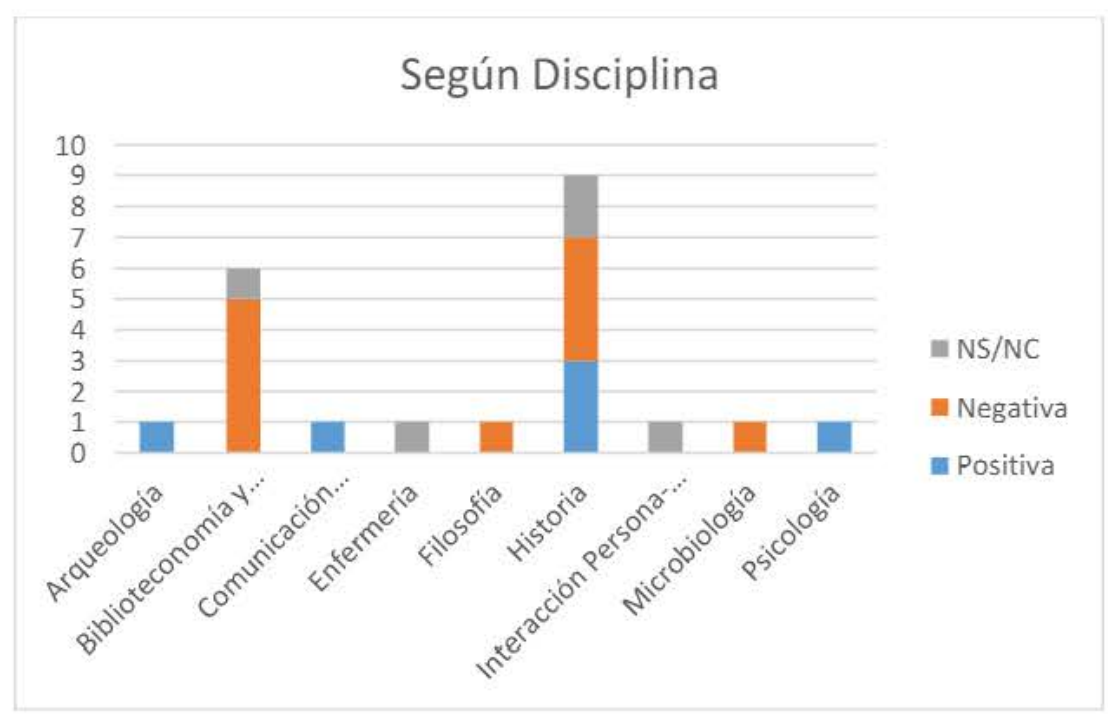

Segmentados por antigüedad en la UB, los resultados muestran una valoración más negativa entre los profesores más nuevos (franja 1991-2012), constatación que se ve confirmada por los resultados por 
edad, donde se aprecia que la insatisfacción aumenta a medida que se reduce la edad de los entrevistados.
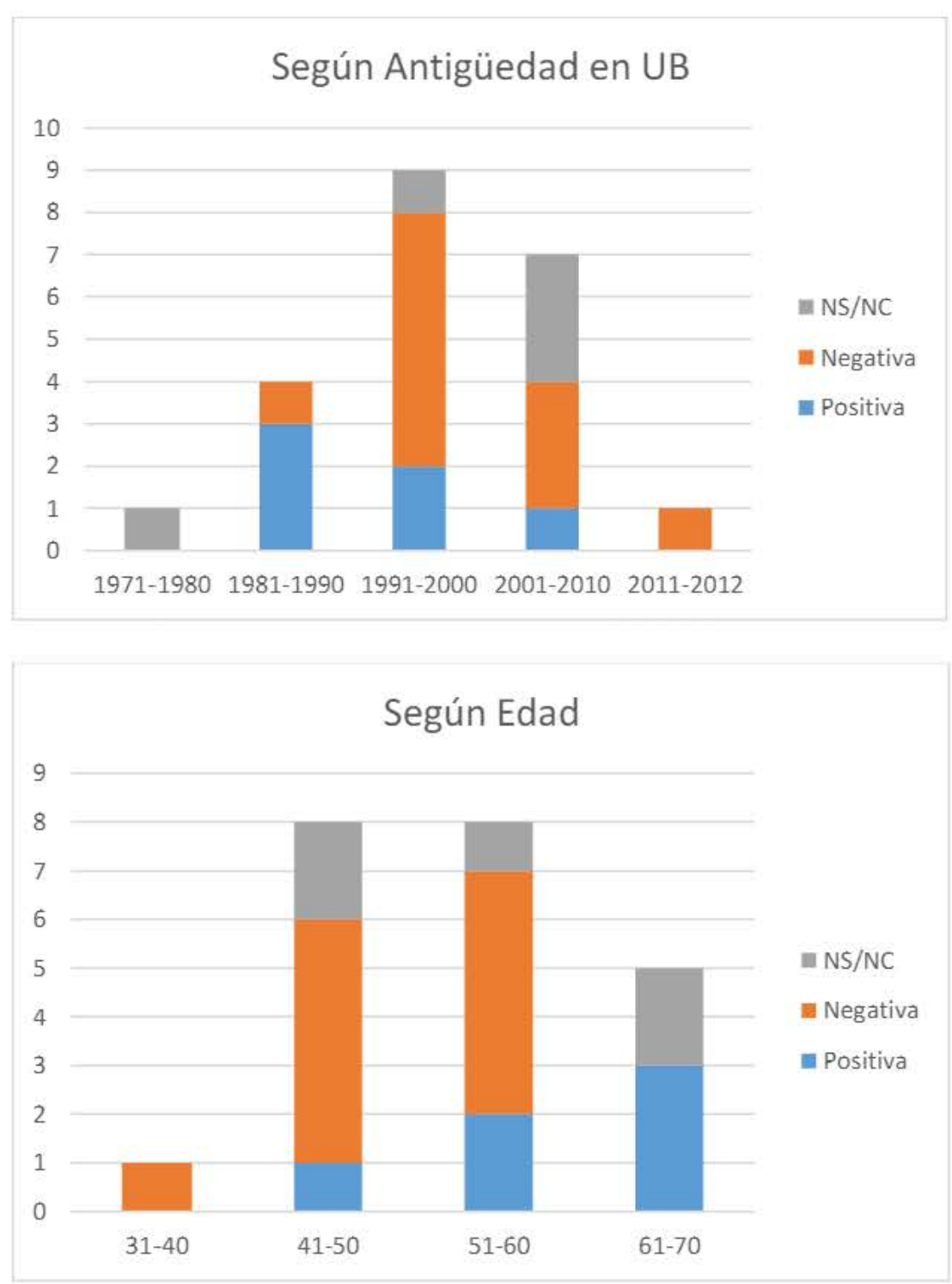

Los datos por dedicación muestran un ligero aumento de la satisfacción en la experiencia de uso de Cercabib entre el profesorado con una mayor actividad docente que investigadora. Aún así, las diferencias con el resto de profesores no son especialmente significativas en este caso. 


\section{Según Dedicación}

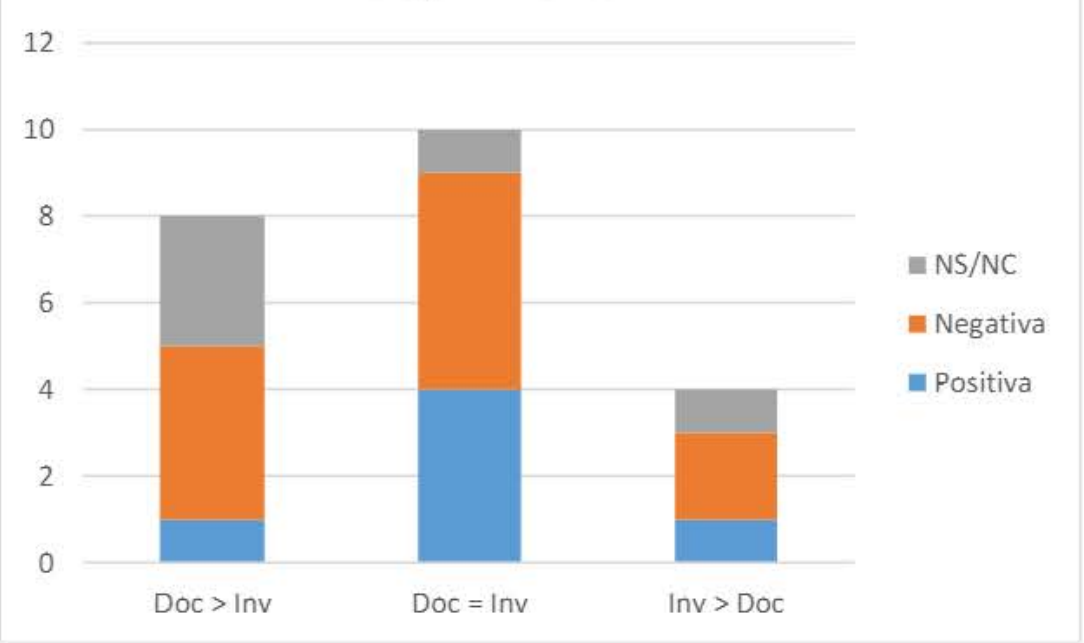

\subsection{Aspectos más positivos y más negativos de Cercabib}

Los aspectos más positivos y negativos de Cercabib expresados por los profesores entrevistados se muestran en las dos tablas siguientes:

\begin{tabular}{|l|c|}
\hline \multicolumn{1}{|c|}{ Aspectos positivos } & Num. Resp. \\
\hline Mucha Información & 5 \\
\hline NS / NC & 5 \\
\hline Nada & 3 \\
\hline Facilidad de uso & 5 \\
\hline Disponibilidad de texto completo & 1 \\
\hline Integración con servicios de la UB & 1 \\
\hline Relación entre recursos & 1 \\
\hline Atractivo visual & 1 \\
\hline
\end{tabular}

\begin{tabular}{|l|c|}
\hline \multicolumn{1}{|c|}{ Aspectos negativos } & Num. Resp \\
\hline Ruido & 9 \\
\hline NS/ NS & 6 \\
\hline Nada & 3 \\
\hline Poco amable & 1 \\
\hline Silencio & 1 \\
\hline Falta información & 1 \\
\hline Visualización deficiente & 1 \\
\hline
\end{tabular}

Como se puede apreciar, en el caso de los aspectos positivos las respuestas son muy variadas, si bien destacan la gran cantidad de información que recupera Cercabib (tanto en número como en tipología documental) y la facilidad de uso. En este último aspecto vale la pena puntualizar que lo que la mayoría del profesorado destacaba como facilidad de uso hacía referencia a la búsqueda simple tipo Google (casilla única) y no tanto otras funcionalidades más especializadas como la búsqueda avanzada o los filtros en la pantalla de resultados.

Como aspecto negativo destaca por encima de todos la gran cantidad de ruido en los resultados de las búsquedas. De una forma u otra, muchos profesores manifestaron su insatisfacción con la ordenación de los resultados de Cercabib y la contrapusieron con los criterios de relevancia de otras herramientas (especialmente Google) que consideran más ajustados a sus necesidades. De hecho, algunos profesores argumentaron esta carencia de Cercabib como uno de los motivos de su poco o nulo uso.

\subsection{Preferencia entre el sistema de búsqueda anterior y Cercabib}

Los resultados de las entrevistas muestran una clara preferencia por el anterior sistema de búsqueda $(50 \%, 11$ de 22). Muchos profesores manifestaron sentirse más cómodos con el anterior servicio del 
CRAI de la UB, y especialmente con el Catàleg de les biblioteques (https://cataleg.ub.edu/). Para ellos el Catàleg era una herramienta donde sabían qué buscaba y dónde lo buscaban. Sabían que los resultados hacían referencia, básicamente, a la colección física de las bibliotecas de la UB; mientras que con Cercabib no saben bien a qué corresponden los resultados, de dónde proceden las referencias, por qué algunas parecen enlazables pero de hecho no lo son, etc. En definitiva, la preferencia por el Catàleg de les biblioteques obedece a menudo tanto a cuestiones funcionales (ruido en los resultados) como a la incomprensión de qué es Cercabib.

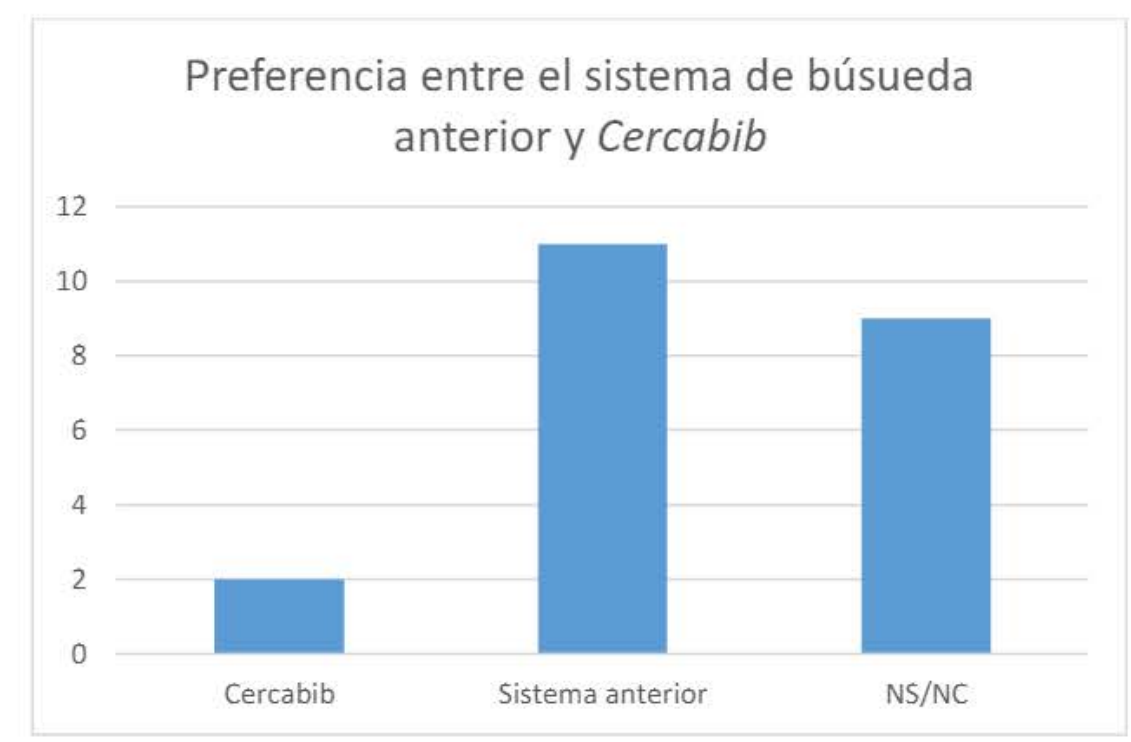

\section{Conclusiones}

Los resultados obtenidos sobre la opinión del profesorado de la Universitat de Barcelona de la nueva herramienta de descubrimiento del CRAI, Cercabib, no difieren substancialmente de los estudios sobre herramientas de descubrimiento expuestos en el apartado "Antecedentes". Como en la mayoría de éstos, la numerosa y diversa información recuperada y la facilidad de uso (búsqueda como Google) son los elementos mejor valorados, mientras los peores son el desconocimiento del alcance de la herramienta, la poca fiabilidad de la ordenación por relevancia y el abrumador número de resultados de búsqueda. Es especialmente relevante, y en cierto sentido preocupante, la mayoritaria preferencia por el anterior sistema de búsqueda.

Las posibles soluciones a estos problemas creemos que pasan, en primer lugar, por el uso de nuevas estrategias de difusión de Cercabib entre el profesorado, puesto que aun reconociendo el encomiable esfuerzo realizado hasta ahora por el personal del CRAI de la UB (sesiones de formación, vídeos, manuales, difusión a través de las redes sociales, etc.), lo cierto es que buena parte de los docentes entrevistados no saben a ciencia cierta qué es Cercabib. En segundo lugar, creemos que hay que mejorar los algoritmos que determinan la ordenación de los resultados de las búsquedas para conseguir una mayor personalización de la recuperación (LIU; LIU; BELKIN, 2019). Sólo de esta manera se podrá superar la sensación de abrumación que impele a muchos profesores a no usar Cercabib y a utilizar herramientas de búsqueda con criterios de relevancia más ajustados (por ejemplo, Google) o de ámbitos temáticos más específicos que garantizan una recuperación más selectiva. 


\section{Anexo}

\section{Información demográfica}

Nombre completo:

Fecha de nacimiento:

Año de inicio de la vinculación con la UB:

Dedicación: docencia (\%), investigación (\%), aproximado

Disciplina:

\section{Cuestionario sobre Cercabib}

a) ¿Te consideras una persona abierta a los cambios tecnológicos?

b) ¿Qué herramientas de búsqueda usas para encontrar información? Google, Google Scholar, Web of Science, Scopus, otros (indica cuáles). ¿Cuál es el primer lugar donde buscas?

c) ¿Utilizas habitualmente los servicios de las bibliotecas de la UB? ¿Con qué frecuencia?

d) ¿Sabes qué es Cercabib?

e) ¿Conoces las principales diferencias entre Cercabib y el anterior sistema de búsqueda del CRAI de la UB? ¿Cuál te gusta más? ¿Por qué?

f) ¿Conoces el alcance de Cercabib (qué puedes encontrar)?

g) ¿Cuántas veces a la semana/mes usas Cercabib?

h) ¿Con qué fines usas Cercabib (apoyo a la docencia, investigación, motivaciones personales, etc.)?

i) ¿Qué tipo de documento búsquedas principalmente en Cercabib (libros, artículos de revistas, ponencias de congresos, material audiovisual, etc.)?

j) ¿Te resulta fácil utilizar Cercabib?

k) ¿Crees que localizas rápidamente la información?

1) ¿Qué opciones de búsqueda usas? ¿Búsqueda simple, avanzada, refinamiento de los resultados?

m) ¿Qué esperarías de un sistema de búsqueda de información de la UB?

n) ¿Qué destacarías como positivo de Cercabib?

o) ¿Qué destacarías como negativo de Cercabib?

\section{Bibliografía}

AHARONY, N.; PREBOR, G. Librarians' and Information Professionals' Perspectives Towards Discovery Tools-An Exploratory Study. The Journal of Academic Librarianship, v. 41, n. 4, p. 429-440, 2015.

ÁVILA-GARCÍA, L.; ORTIZ-REPISO, V.; RODRÍGUEZ-MATEOS, D. Herramientas de descubrimiento: ¿una ventanilla única? Revista Espanola de Documentacion Cientifica, v. 38, n. 1, p. 1-17, 2015.

DALAL, H. A.; KIMURA, A. K.; HOFMANN, M. A. Searching in the wild: Observing informationseeking behavior in a discovery tool. Proceedings of the ACRL, p. 668-675, 2015.

FAWLEY, N.; KRYSAK, N. Learning to Love Your Discovery Tool: Strategies for Integrating a Discovery Tool in Face-To-Face, Synchronous, and Asynchronous Instruction. Public Services Quarterly, v. 10, n. 4, p. 283-301, 2 out. 2014.

GONZÁLEZ-TERUEL, A.; BARRIOS CERREJÓN, M. T. Métodos y técnicas para la 
investigación del comportamiento informacional: fundamentos y nuevos desarrollos. Gijón: Trea, 2012.

LIU, J.; LIU, C.; BELKIN, N. J. Personalization in text information retrieval: A survey. Journal of the Association for Information Science and Technology, v. 0, n. 0, 6 maio 2019.

NICHOLS, A. F. et al. What Does it Take to Make Discovery a Success?: A Survey of Discovery Tool Adoption, Instruction, and Evaluation Among Academic Libraries. Journal of Web Librarianship, v. 11, n. 2, p. 85-104, 2017.

SHAPIRO, S. Academic Librarians, Information Overload, and the Tao of Discovery. The Journal of Academic Librarianship, v. 44, n. 5, p. 671-673, 2018.

WELLS, D.; RICHARDSON, C. How do library clients use discovery systems. LIANZA Conference. Anais...2014 